\title{
Design optimization application in accordance with product and process requirements
}

\author{
A. Del Prete, D. Mazzotta \& A. Anglani \\ Department of Engineering Innovation, Lecce University, Italy
}

\begin{abstract}
Structural optimization techniques are a well-known approach for improving product performances. Often, optimization procedures do not include manufacturing constraints arising from corporate technologies. This aspect becomes a disadvantage in the design review phase when the final product release is a trade-off between optimization results and manufacturing constraints. This paper describes a specific new approach, which considers product/process guidelines an input/output data in the optimization phase. The study case is represented by a high performance aeronautic seat structure having as mission profiles the SAE-AS Standard, in order to demonstrate occupant protection when a seat/occupant/restraint system is subjected to statically applied ultimate loads and to dynamic impact test conditions. The authors' aim, in accordance with standards' requirements, is to achieve a final design based on an optimized structural solution for the chosen process technologies, taking into account the low volume production and typical attitude of the aeronautical industry. The presented study case offers the proper reference in order to extend this methodology to more complex structural applications.
\end{abstract}

Keywords: topology optimization, manufacturing constraints, product/process guidelines.

\section{Introduction}

The ability to introduce new products in a shorter lead time, to new and existing markets is a dominant feature of companies operating in a concurrent engineering environment $[1,2]$. Concurrent engineering is also a comprehensive approach to production of goods and integrates the design process with materials, manufacturing methods, process planning, assembly testing and quality control. Designers must be able to assess the impact of design 
modifications on manufacturing processes selection, assembly, inspection, tools and dies, and product cost. It is essential to establish quantitative relationships in order to optimize the design for ease of manufacturing and to assembly at minimum product cost (also called producibility). Computer-aided techniques have become indispensable to such analysis [3]. In this paper authors present a new approach to redesign an existing rotorcraft comfort seat structure; product requirements and manufacturing constraints are used respectively as input and output to structural optimization phase. The proposed design solution for this high comfort and performances rotorcraft seat have as performances references the SAE-AS8049 Standard ones [4].

\section{Proposed design procedure}

The rotorcraft seat can be considered as composed by four main subassemblies: base, chair seat, chair back, mechanisms (Figure 1(a)).

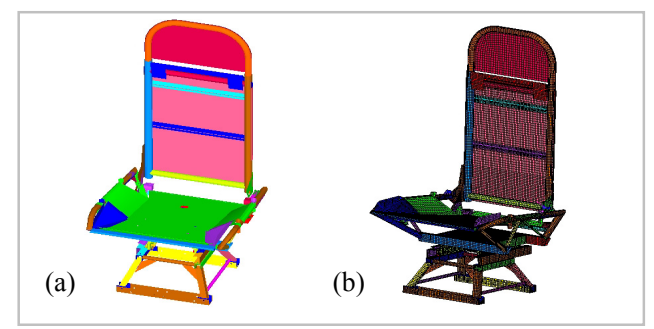

Figure 1: Existing design: (a) parametric models; (b) finite element models.

An appropriate finite element model (Figure 1(b)) has been developed in order to perform a static and dynamic analysis having as reference the SAE AS8049 REV B.

In this paper, the authors illustrate the application of the developed methodology and the capabilities of the applied tools to a seat subpart only: the base (Figure 2).

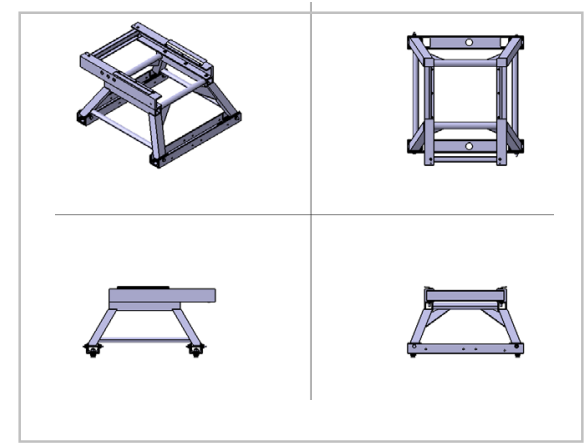

Figure 2: $\quad$ Existing seat base. 
The suggested procedure is made by the following phases: product requirements definition, design optimization, process guidelines and manufacturing constraints definition, new proposed design, product validation tests (e.g. dynamic impact tests, digital mock up), design delivery. The proposed procedure is shown in the following flow diagram (Figure 3).

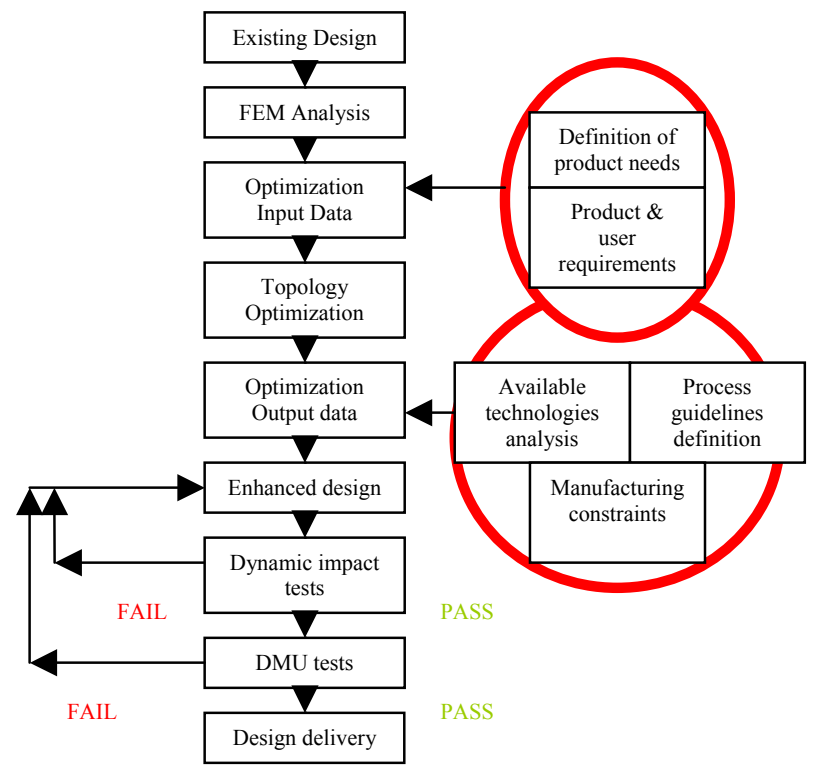

Figure 3: Design procedure.

For the other seat subparts, the base has been improved using the illustrated procedure.

\section{New design definition in accordance with the proposed procedure}

The existing base is an unassemblable multi welded component. Starting from the original base model, the authors carry out the scheduled steps.

\subsection{Step 1: Product and user requirements definition}

Product requirements are characteristics given by the application field of the studied part, user requirements are, as they are defined, given by end users' needs. The whole development process must be user-oriented if the user requirements are to be fulfilled by the final product. Product and user requirements' definition has an enormous influence on the entire product/process development phase and on the product success in its market of reference, in this 
specific case they are used as input data for the topology optimization phase. The chosen requirements for the base study case are:

- dimensional characteristics: the considered structure is a VIP aeronautical seat, for this reason occupant can choose his preferred position through the degrees of freedom given by the seat mechanisms (horizontal translation, chair back inclination and seat rotation). This need can be easily translated in a design requirement in order to reduce as much as possible the volume of the fixed part which has to be connected with the helicopter floor. In this case the dimensions of the original design are considered. This assumption is important for the design space definition;

- weight: weight reduction is one of the added values for a typical aeronautical product. In the present activity topology optimization is used in order to reduce the structure weight which is considered a constraint of the optimization problem through a specific function defined as massfrac (Massfrac $=$ Component Initial Mass/Design Space Mass);

- assemblability criteria: the chance to have the easiest possible assembly operation is taken into account; this decision has influence in the optimization phase for the design space geometry definition.

\subsection{Step 2: Optimization}

'Optimization' is about selecting the best option from a range of possible choices. It is natural to consider it when designing a new product. Design optimization aims to give a new better design compared with the initial structure. The algorithms dedicated to this task are used for searching the best solution within a given design space. In order to achieve an incisive design optimization, in this case, the authors apply a topological optimization [6] through the usage of a specific software tool: OptiStruct ${ }^{\mathbb{B}}$. It solves the following structural optimization problem:

Objective: Minimize $W(x)$

Constraints: $g(x)-g^{u} \leq 0$

Design Variables: $x^{L} \leq x \leq x^{u}$

The objective function $W$ and the constraint function $g$ are structural responses obtained from a finite element analysis. The selection of the vector of design variables, $x$, depends on the type of optimization being performed.

The method used is an iterative procedure known as "local approximation method". This method determines the solution of the optimization problem using the following steps:

- $\quad$ analysis of the physical problem using finite elements;

- $\quad$ convergence test, whether or not the convergence is achieved;

- $\quad$ design sensitivity analysis;

- $\quad$ solution of an approximate optimization problem formulated using the sensitivity information;

- $\quad$ back to the first step. 
Optistruct solves topological optimization problems using either a homogenization or a density method [6]. Topology Optimization is a mathematical technique that produces an optimized shape and material distribution for a structure within a given package space. By discretizing the domain into a finite element mesh, it calculates material properties for each element. The algorithm alters the material distribution to optimize the userdefined objective under given constraints. Many of the most common structural responses are available as objective or constraint functions.

In this specific case, the subparts, which are objects of the topological optimization are: chair back, base and chair seat considering the most onerous load situation with the double purpose of stiffness increase and weight reduction (Table 1). This approach allows one to consider these three different parts of the structure as independent. This can be considered an advantage from the manufacturing point of view because their production phases can be considered parallel.

Table 1: $\quad$ Optimization loads.

\begin{tabular}{|c|c|c|c|c|c|}
\hline & Rearward & Downward & Forward & Upward & $\begin{array}{c}\text { Sideward } \\
\text { DX }\end{array}$ \\
\hline $\mathrm{CB}$ & $\mathrm{X}$ & & & & \\
\hline $\mathrm{B}$ & & $\mathrm{X}$ & $\mathrm{X}$ & & $\mathrm{X}$ \\
\hline $\mathrm{CS}$ & & $\mathrm{X}$ & $\mathrm{X}$ & & $\mathrm{X}$ \\
\hline
\end{tabular}

In this case two different responses are considered: compliance and massfrac. The compliance is calculated as:

$$
\begin{aligned}
& C=1 / 2 u^{T} f \text { with } \quad K u=f \\
& C=1 / 2 u^{T} K u=1 / 2 \int \varepsilon^{T} \sigma d V
\end{aligned}
$$

It represents the deformation energy of the structure and it can be considered as the inverse of the structure stiffness.

The massfrac response is calculated as: Massfrac $=$ Component Initial Mass/Design Space Mass.

A Design Space volume and a Non Design space volume are defined for each part subjected to optimization

(a)

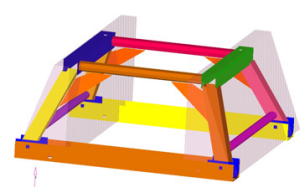

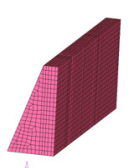

$\operatorname{lin}_{4}$

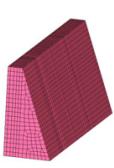

(b)

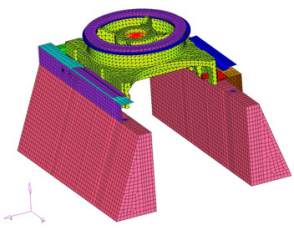

Figure 4: $\quad$ FE model and design space in: (a) SOL1; (b) SOL2.

For the base structure the considered loading conditions are: forward, downward, sideward $d x$. Different design spaces are used during the 
optimization phase in order to evaluate the problem sensitivity to different "volumetric" conditions. Topology optimization has been performed for each loading condition. The obtained solutions were considered in order to evaluate a possible solution for the new structure (Figure 4).

The first option (SOL1) it is defined having a design space (Figure 4(a)) including the lateral volume of the actual solution without considering the volume between the two sides according with the linear static analysis results of the original structure.

Then a reduced model of the structure (SOL2) is considered by applying loading conditions directly on the central seat frame and its rails (Figure 4(b)).

The last considered option (SOL3) is represented by a design space defined by the entire volume included in the original base frame (Figure 5) in order to verify the absence of contribution of the inner part for higher performance topology solutions.

Table 2 reports the used parameters for the optimization set-up of the different models following the illustrated procedure.

In the following figures (Figures 6-8) some of the obtained results are reported.
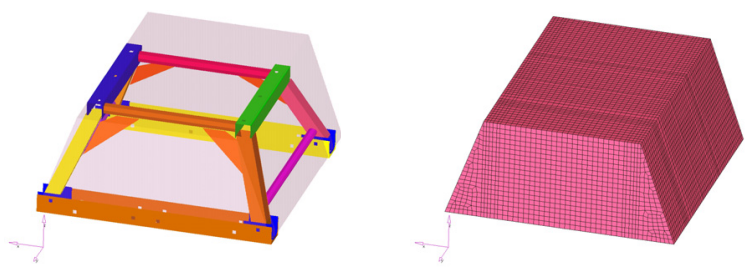

Figure 5: $\quad$ FE model and design space in OPTI_BASE3.

Table 2: $\quad$ Optimization loads.

\begin{tabular}{|c|c|c|c|c|}
\hline Simulation & $\begin{array}{c}\text { D constrain } \\
\text { (massfrac) }\end{array}$ & $\begin{array}{c}\text { Objective Function } \\
\text { Min compliance }\end{array}$ & $\begin{array}{c}\text { MinDim } \\
(\mathrm{mm})\end{array}$ & $\begin{array}{c}\text { Technological } \\
\text { constrain }\end{array}$ \\
\hline SOL1 & 0.08 & $\mathrm{X}$ & 29 & Symmetry (x) \\
\hline SOL2 & 0.08 & $\mathrm{X}$ & 29 & Symmetry (x) \\
\hline SOL3 & 0.05 & $\mathrm{X}$ & 29 & Symmetry (x) \\
\hline
\end{tabular}

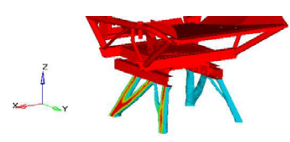

(a)

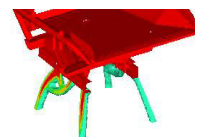

(b)

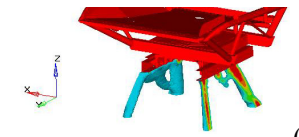

(c)

Figure 6: SOL1 Material density distribution: (a) downward (density 0.33); (b) forward (density 0.38); (c) sideward dx (density 0.25 ). 


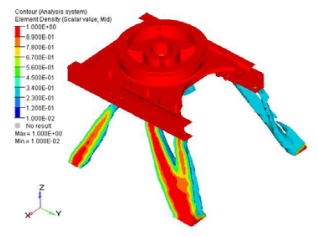

Figure 7: SOL2 Material density distribution with matched loads (density 0.32).

As can be evaluated by Figure 8, even if the design space (SOL3) fulfilled the original base volume no cross members would be defined by the optimizer in accordance with the solutions found with SOL1 and SOL2.

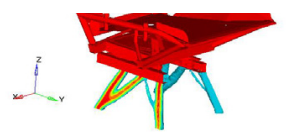

(a)

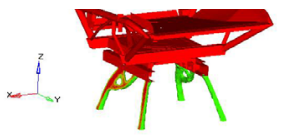

(b)

Figure 8: SOL3 Material density distribution: (a) downward (density 0.25); (b) forward (density 0.50 ).

\subsection{Step 3: Process requirements and manufacturing constraints definition}

The obtained geometric results are evaluated in accordance with process and manufacturing requirements which are defined having as reference.

- low production volumes: due to the fact that the reference market is a niche one, the production rates are characterized by low volumes. Authors have oriented their interest to technological options which require low investments. Welded connections have been avoided; it has been preferred to use components obtained by metal cutting.

- assemblability criteria: in order to guarantee an easier management of the assembly phase and then easier assembling/unassembling operations for eventual part substitutions, a driving criteria is defined in order to respect this process requirement. The usage of the metal cutting technology demonstrates its advantages also in this case.

- available technologies: For technical considerations about metal cutting, this is considered the chosen technology.

\subsection{Step 4: Enhanced design}

Considering the shape (OSSmooth), given back as optimization output, in conjunction with established process requirements and manufacturing constraints, the authors propose a new design and its relative constructive drawing (Figure 9).

Therefore, taking in account the illustrated step 3, the enhanced design is made by two machined components only, which can be obtained by relatively simple cutting operations (contour machining 21/2 axis), and made in small quantities because they do not require considerable investment costs. 


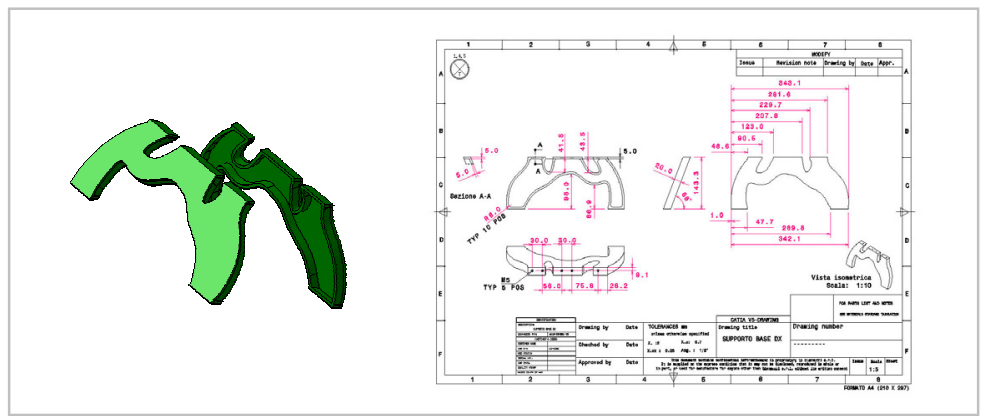

Figure 9: Industrial design of the optimized shape ready for production.

\subsection{Step 5: Verification tests}

The obtained design solution for the base structure is integrated, as the ones obtained for the other parts in the new seat model for which a FEM is developed. The optimized structure passed all the verification tests defined in accordance with SAE AS8049 REV B Standards. As reference, in this section dynamic tests results are reported for the "Forward" and "Down" dynamic conditions (Figure 10). The FEM model for the explicit analysis is completed with an Hybrid III 50\% dummy model.

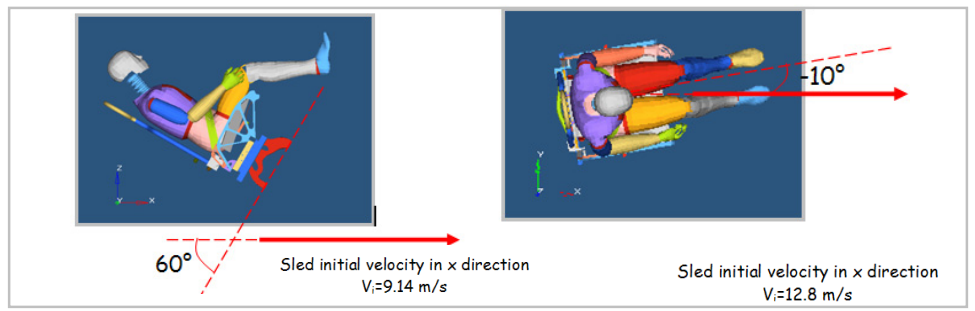

Figure 10: Down and forward tests set-up conditions.

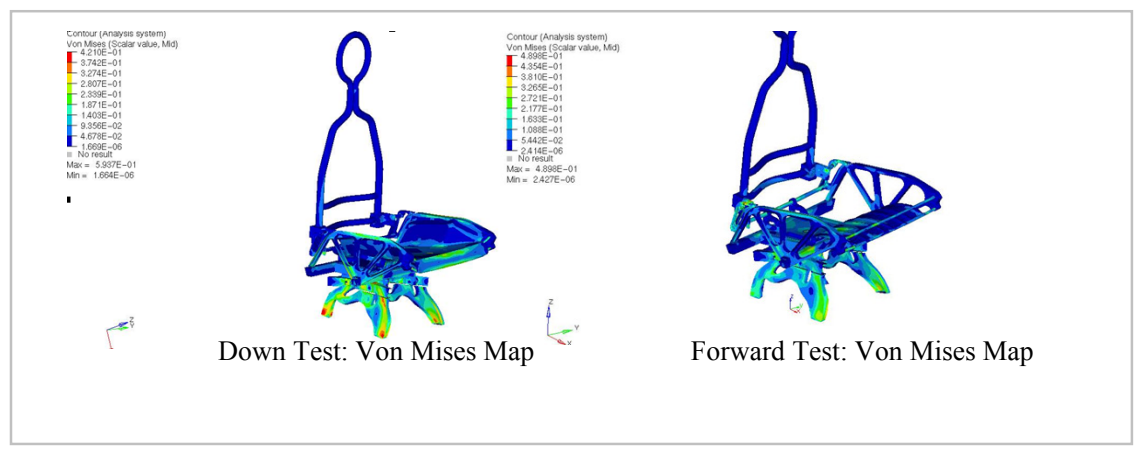

Figure 11: $\quad$ Structural performance in test conditions $(\mathrm{GPa})$. 
In both test conditions the structural performance and the occupant parameters are within the range defined by the SAE AS Standards. Figure 11, as example, shows the stress distribution for the two considered conditions.

In this methodology, the authors propose a Digital Mock Up phase, as verification test. The current avalaibility of Computer Aided instruments allow many verification analyis with more than one interaction with the previous steps.

\subsection{Step 6: Design delivery and prototype carrying out}

Good feed back, arising from the previous step of the developed procedure, permits us to deliberate a final design. A comparison between the old and the new design is shown in Figure 12.

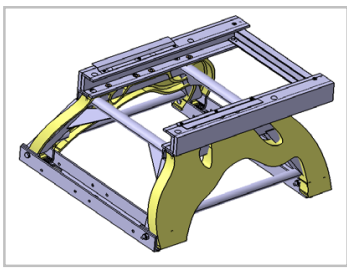

Figure 12: Comparison between the old (grey) and the new (yellow) base.
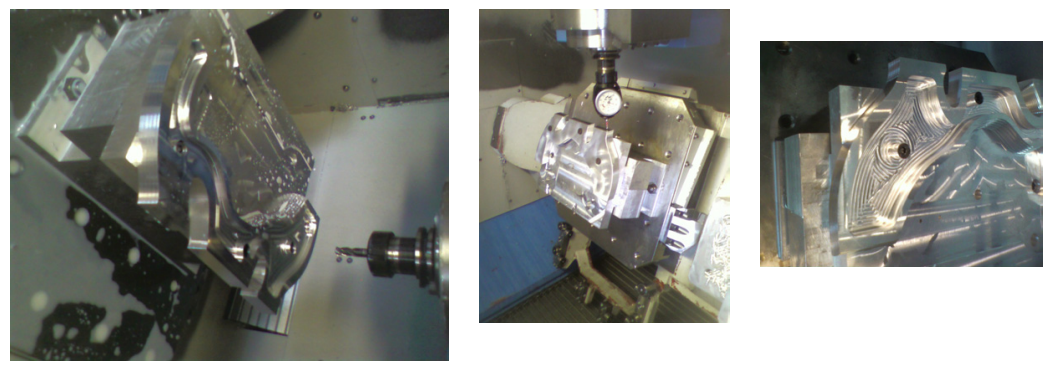

Figure 13: Machining phases.

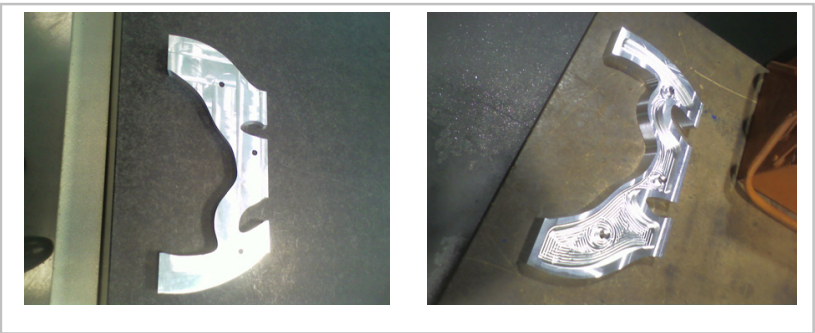

Figure 14: Obtained component. 
As a final part of this application, but no less important, it is useful to describe some aspects related to the machining phases necessary for the prototype production (Figure 13) and to obtain the physical component (Figure 14). Small modifications were required like the addition of three holes to ensure an effective fixing between workpiece and machine tool.

\section{Conclusions}

The carried out activity allows one to verify, practically, the real benefit arising from proposed procedure. The authors intend to proceed in this direction developing a general effectiveness and at the same time to verify interaction between used software and customized applications (process automation). In this way the product/process designer could be focused on the engineering problem and not on the way of how to fit it with the available CAX technologies.

\section{References}

[1] Lettice, F., Palminder, S., Stephen, E. A workbook-based methodology for implementing concurrent engineering, International Journal of Industrial Ergonomics, 16, pp. 339-351, 1995.

[2] Edwards, K.L. Towards more strategic product design for manufacture and assembly: priorities for concurrent engineering, Materials and Design, 23, pp. 651-656, 2002.

[3] Kalpakjian, S. Manufacturing Processes for Engineering Materials, Addison Wesley, pp. 11-13, 1991.

[4] SAE AS8049 rev. B Performance Standard for Seats in Civil Rotorcraft, Transport Aircraft, and General Aviation Aircraft.

[5] Schramm, U., Thomas, H.L., Zhou, M., and Voth, B. Topology Optimization with Altair OptiStruct, Proceedings of the Optimization in Industry II Conference, Banff, CAN, 1999.

[6] Bendsøe, M., and Kikuchi, N. Generating Optimal Topologies in Optimal Design using a Homogenization Method, Computer Methods in Applied Mechanics and Engineering, 71, 197-224, 1988.

[7] Goldberg, D.E. Genetic algorithms in search, optimisation and machine learning, Addison-Wesley, 1988.

[8] SAE ARP5526 Aircraft Seat Design Guidance and Clarifications. 